\title{
Answer to the Letter to the Editor of Miao Yu et al. concerning "Is L5-S1 motion segment different from the rest? A radiographic kinematic assessment of 72 patients with chronic low back pain" by AB Sabnis et al. (Eur. Spine J; 27(5):1127-1135)
}

\author{
Ashutosh B. Sabnis ${ }^{1}$. Uphar Chamoli ${ }^{1}$. Ashish D. Diwan ${ }^{1}$ \\ Received: 15 June 2018 / Accepted: 16 June 2018 / Published online: 22 June 2018 \\ (c) Springer-Verlag GmbH Germany, part of Springer Nature 2018
}

We would like to thank the authors of the letter for showing interest in our work. Undoubtedly, the disc height and spinal alignment will be altered in the supine position compared with the standing position, and it will be useful to evaluate the relationship between kinematics and degenerative changes in the standing position. An upright magnetic resonance (MR) scanner allows the patient to be scanned in the standing and several other weight-bearing positions [1]. However, currently available upright MR scanners generate relatively low magnetic field strengths ranging from 0.2 to 0.6 Tesla, and the acquired images are characterised by lower resolution and lower signal-to-noise ratios than the high-field recumbent MR scanners [2, 3]. Since our study was retrospective in nature, this option was not available to us.

The index-level mid-sagittal plane disc height in our study was assessed by making comparisons with the cephalad disc height. Based on our disc degeneration scoring protocols, we posit that disc-height-loss scores will not change significantly between a recumbent and an upright MR. However, as pointed out by the authors, the assessment would be challenging in patients with multilevel disc pathologies. The absence of a control group comprising asymptomatic subjects without any degenerative changes in the lumbar spine is a recognised limitation of this study.

It has been reported previously that disc degeneration, sagittal alignment, and segmental mobility in the lumbar spine have a reciprocal influence on each other [4]. We agree that the influence of different types of lumbar sagittal

Uphar Chamoli

u.chamoli@unsw.edu.au

1 Spine Service, Department of Orthopaedic Surgery, St. George and Sutherland Clinical School, University of New South Wales, Kogarah, Sydney, NSW 2217, Australia alignment (kyphotic, straight, normal lordotic, hyperlordotic), spino-pelvic alignment (balanced and retroverted pelvis), and knee flexion on lumbar segmental kinematics needs further investigation.

Whilst we continue our efforts to better understand the relationship between segmental kinematics and degenerative changes in the lumbar spine, we look forward to seeing welldesigned prospective studies from other authors in this vein, addressing some of the above-mentioned issues.

\section{Compliance with ethical standards}

Conflict of interest The authors declare no conflict of interest related to this work.

\section{References}

1. Botchu R, Bharath A, Davies AM, Butt S, James SL (2018) Current concept in upright spinal MRI. Eur Spine J 27:987-993. https ://doi.org/10.1007/s00586-017-5304-3

2. Hansen BB, Hansen P, Christensen AF, Trampedach C, Rasti Z, Bliddal H, Boesen M (2018) Reliability of standing weightbearing $(0.25 \mathrm{~T}) \mathrm{MR}$ imaging findings and positional changes in the lumbar spine. Skelet Radiol 47:25-35. https://doi.org/10.1007/ s00256-017-2746-y

3. Jinkins JR, Dworkin JS, Damadian RV (2005) Upright, weightbearing, dynamic-kinetic MRI of the spine: initial results. Eur Radiol 15:1815-1825. https://doi.org/10.1007/s00330-005-2666-4

4. Keorochana G, Taghavi CE, Lee KB, Yoo JH, Liao JC, Fei Z, Wang JC (2011) Effect of sagittal alignment on kinematic changes and degree of disc degeneration in the lumbar spine: an analysis using positional MRI. Spine 36:893-898. https://doi.org/10.1097/ BRS.0b013e3181f4d212 